\title{
Multi-Method 3D Characterization of Different Tissue Types in Healing Bone
}

Victoria Schemenz ${ }^{1,2}$, Fereshteh Falah Chamasemani ${ }^{3}$, André Gjardy ${ }^{1}$, Paul Roschger ${ }^{4}$, Peter Fratzl ${ }^{1}$, Lukas Helfen ${ }^{5}$, Richard Weinkamer ${ }^{1}$, Roland Brunner ${ }^{3}$, Bettina M. Willie ${ }^{6}$ and Wolfgang Wagermaier $^{1}$

${ }^{1 .}$ Max Planck Institute of Colloids and Interfaces, Department of Biomaterials, Research Campus Golm, Potsdam, Germany.

2. Berlin-Brandenburg School for Regenerative Therapies (BSRT), Berlin, Germany.

3. Materials Center Leoben Forschung GmbH, Leoben, Austria.

4. Ludwig Boltzmann Institute of Osteology at Hanusch Hospital of WGKK and AUVA Trauma Centre Meidling, 1st Medical Department, Hanusch Hospital, Vienna, Austria

5. European Synchrotron Radiation Facility (ESRF), Grenoble, France.

6. Research Centre, Shriners Hospitals for Children-Canada, Department of Pediatric Surgery, McGill University, Montreal, Quebec, Canada.

Bone has the ability to regenerate its structure and function after fracture. Moreover, the repair of bone fractures recapitulates skeletal development and therefore healing bone is an ideal model system to study correlations between the architecture of the lacuno-canalicular network (LCN) and the surrounding bone matrix in different tissue types. At the site of fracture, first a soft tissue is formed which subsequently mineralizes and mechanically stabilizes the bone. The first formed mineralized tissue exhibits a rather low degree of structural organization at the fibrillar level and is later augmented by a more lamellar organized tissue. During mechanically induced remodelling the callus tissue is gradually replaced by bone which resembles the original bone structure of the cortex with a high degree of organization. This process is equal to the lifelong interplay between bone resorption by osteoclasts and bone formation by osteoblasts. Osteocytes are embedded in the extra cellular matrix (ECM) and orchestrate the remodeling process via fluid flow in the lacuno-canalicular network (LCN) and likely contribute directly to mineral homeostasis. To further elucidate the functionality of osteocytes, we apply a correlative analysis of ECM properties and LCN architecture within the same bone volume.

Confocal laser scanning microscopy and high resolution synchrotron $\mu \mathrm{CT}$ measurements are used for a three-dimensional characterization of the LCN. The mineral content of the sample surfaces is measured by quantitative backscattered electron imaging (qBEI) and the mineral particle characteristics in the ECM by synchrotron scanning small/wide angle X-ray scattering (SAXS/WAXS). From the synchrotron $\mu \mathrm{CT}$ data (Fig. 1) the following parameters are analyzed to characterize the osteocyte lacunae in cortex and callus tissue and in particular at the interface region in between: (1) diameter, (2) volume, (3) oblateness, (4) orientation, (5) closest distance and (6) lacunar density. In order to determine those parameters we perform pre-processing, thresholding and segmentation as well as post-processing of the image data by using Python and Avizo for vizualisation (Fig. 1).

Data about the LCN and ECM in healing bone do not only allow a distinction between cortex, cartilage and callus, but also between different bone types within the callus. Callus bone exhibited on average larger and less elongated lacunae as well as higher lacunae density compared to cortical lamellar bone. Within the callus, woven-like bone areas showed thicker and less organized mineral particles in comparison to newly formed callus lamellar bone. Overall, we identified correlations between osteocyte network architecture and mineral particle characteristics in all tissue types. 


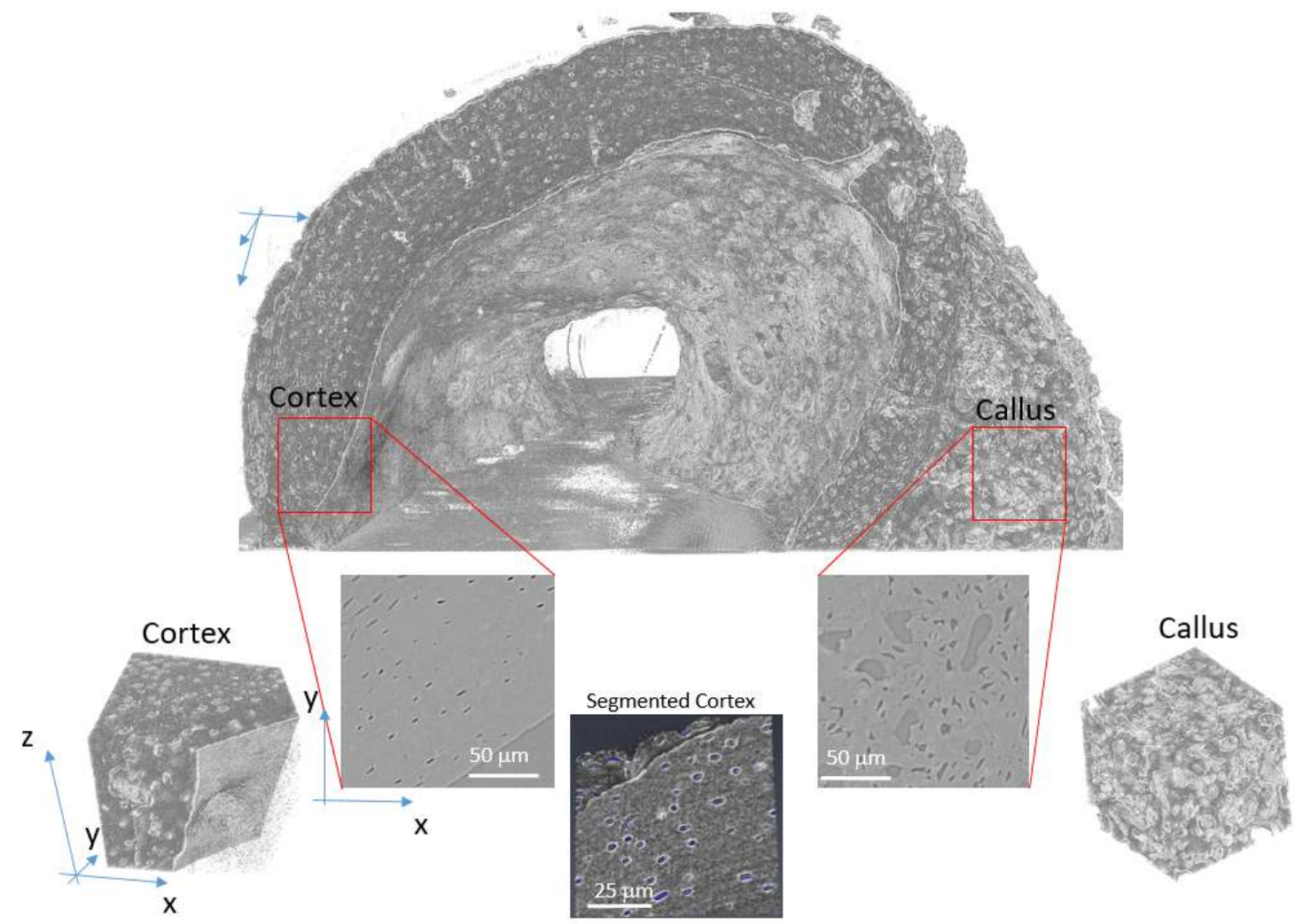

Figure 1. 3D representation of the bone using synchrotron tomography. Two different regions are shown and labled as cortex- and callus-region. Slice image (x-y-plane) of the callus and cortex region (below) as well as the corresponding 3D volume. Middle: segmented lacunae (blue) and bone matrix (grey) for the cortex. 